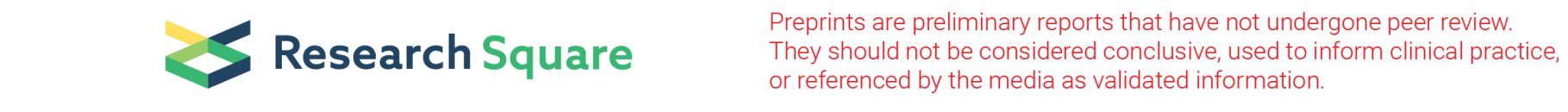

\title{
General Electrochemical Minisci Alkylation of N- Heteroarenes with Alkyl Halides
}

Jose Aleman ( $\nabla$ jose.aleman@uam.es )

Autonomous University of Madrid https://orcid.org/0000-0002-9154-4067

\section{Article}

Keywords:

Posted Date: January 12th, 2022

DOI: https://doi.org/10.21203/rs.3.rs-1229384/v1

License: (a) (i) This work is licensed under a Creative Commons Attribution 4.0 International License. Read Full License 


\section{Abstract}

Herein, we report, for the first time, a general, facile and environmentally friendly Minisci-type alkylation of $N$-heteroarenes under simple and straightforward electrochemical conditions using widely available alkyl halides as radical precursors. Primary, secondary and tertiary alkyl radicals have shown to be efficiently generated and coupled with a large variety of $N$-heteroarenes. The method presents a very high functional group tolerance, including various heterocyclic-based natural products, which highlights the robustness of the methodology. This applicability has been further proved in the synthesis of various interesting biologically valuable building blocks. In addition, we have proposed a mechanism based on different proofs and electrochemical evidences.

\section{Introduction}

$N$-heterocycles have attracted the attention of organic chemists over the years as they are versatile intermediates in organic synthesis and prevalent structures in natural and synthetic products with a wide spectrum of biological properties.' Indeed, more than $85 \%$ of all biologically active compounds are heterocycles, highlighting their importance in organic and medicinal chemistry. ${ }^{1}$ Due to their relevance, the development of straightforward functionalizations of heterocyclic structures has been a focal point in organic chemistry research, with special emphasis on strategies capable of replacing $\mathrm{C}-\mathrm{H}$ bonds with new and exciting functionalities in a single, direct and selective operation.' In this context, the venerable Minisci reaction stands as a powerful and appealing synthetic tool for the direct and rapid modification of heteroaromatic units. Based on their innate reactivity, addition of a nucleophilic carbon radical species followed by a $\mathrm{H}$ atom loss provides the desired $\mathrm{C}-\mathrm{H}$ functionalized derivatives; a reaction pathway which can afford a quick and efficient increase in molecular complexity.' As a consequence of the importance of this challenge, a plethora of research groups have focused their efforts on developing new Minisci-type alkylation methodologies. ${ }^{4,5}$ Spurred by the resurgence in the field of photocatalysis, several reports have surfaced based on visible-light-mediated Minisci-type alkylations using a variety of radical precursors including, for example, peroxides, alcohols, boronic acids, alkyltrifluoroborates, carboxylic acids"', redox-

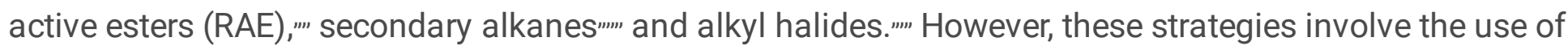
expensive metal-based photocatalysts, excess of additives (silanes, oxidants, surfactants, etc), inert atmosphere and long reaction times that hamper their broad applicability.

Among the multiple strategies based on the single-electron activation of organic substrates, electrochemistry is quickly becoming one of the most popular avenues to access radical intermediates. The use of simple electrons as reagents through application of an electrical current offers the possibility to promote redox events and carry out reactions under mild conditions with enhanced atom economy."m In this context, electrochemical Minisci-type processes have recently begun to attract considerable attention employing different kinds of alkyl radical precursors, such as sulfinates, RAE, and carbazates (Figure 1A). In this context, alkyl halides are of particular interest when looking at their radical precursor potential since they are readily available and inexpensive. Despite the great interest of including alkyl halides in the 
Minisci reaction electrochemical portfolio, this objective has not been accomplished. In fact, the electroreduction of radical precursors to engage in the formation of new $\mathrm{C}-\mathrm{C}$ bonds are now being pursued and studied by various research groups $39,40,41_{\text {"m }}$ as they allow the development of new methodologies with high generality and functional group tolerance when compared with less indulgent oxidative reaction conditions but never applied in Minisci-type alkylation processes. Thus, we envisioned that the development of a facile and operationally simple Minisci-alkylation via electroreduction of readily available alkyl halides would be highly appealing, which may also provide new opportunities using this straightforward approach to generate carbon-centered radicals, as electrochemical reduction of alkyl halides requires highly reducing potentials which has hampered its general application caused by the incompatibility of several functional groups with such reaction conditions. ${ }^{47}$,

Herein, we describe a Minisci-type alkylation using alkyl halides under simple, mild and easy-to-handle electrochemical conditions (Figure 1B). The electrochemically generated aliphatic radicals efficiently engage with nitrogen-based heteroarenes in the presence of a Brønsted acid as sole promoter.

\section{Results}

Optimization of the model reaction. We began our investigations by studying the reaction of 4methylquinoline (1a) as model substrate with cyclohexyl iodide (2a) as radical precursor (Table 1). By using diphenyl phosphate (PA), $\mathrm{NH}_{4} \mathrm{PF}_{6}$ as electrolyte under $10 \mathrm{~mA}$ constant current for 120 mins in an undivided cell, the alkylated quinoline derivative $3 a$ was isolated in excellent yield (92\%, entry 1 ). Operationally, the setup uses a simple commercial potentiostat, without any of the ordinary precautions required in radical chemistry, such as meticulous exclusion of $\mathrm{O}_{2}$ or water, since the reaction takes place in a mixture of THF: $\mathrm{H}_{2} \mathrm{O}$ under air. For the development of a synthetically useful electrochemical Miniscitype alkylation, various parameters such as the additive or promoter, solvent, electrolyte, electrode material and electrochemical parameters have been studied and summarized in Table 1 (see Supplementary Information for details). When the reaction was stopped after $42 \mathrm{mins}(2.4 \mathrm{~F} / \mathrm{mol}), 73 \%$ conversion of the desired alkylated heteroarene was obtained (entry 2). In presence of other acids such as trifluoroacetic acid (TFA) or para-toluenesulfonic acid $(p T s O H)$ the reaction took place less efficiently and lower reactivities were observed (entries 4 and 5), which demonstrates the special capability of the PA to carry out the activation of quinoline derivatives. ${ }^{17}$ Different solvent systems commonly used under electrochemical reaction conditions (entries 6-9, see Supplementary Information for details) were then evaluated, showing that $\mathrm{H}_{2} \mathrm{O}$ as co-solvent was necessary to competently perform the alkylation. Modification of the electrolyte showed that the use of a Brønsted acid-based electrolyte had a considerable effect on the reaction as it might be also involved in the activation of the heterocycle (entries 10 and 11). The electrode material selection had a great impact on the reaction as well. Thus, $\mathrm{Zn}$ or carbon-based electrodes (RVC) cathodes did not promote the reaction (entries 12 and 13). Finally, the reaction did not take place in absence of electrical current (entry 14). 
Substrate Scope. Once the reaction conditions had been optimized, a variety of alkyl iodides were tested under the electrochemical reaction conditions in an undivided cell with a readily available RVC anode and a nickel foam cathode. The initial aim of this study was to evaluate the generality of the system using 4methylquinoline as model substrate (Table 2). This exploration demonstrated that secondary alkyl radical precursors efficiently performed the alkylation in good yields (3a-3f). Notably, secondary alkyl iodides bearing heteroatoms were very well tolerated (3c, $\mathbf{3 d}$ and $\mathbf{3} \mathbf{f})$. A sterically encumbered tertiary alkyl radical also led to the desired alkylated quinoline with very good yield $(\mathbf{3 g})$. Furthermore, primary alkyl radicals were competently generated under the electrochemical conditions presented and furnished the desired 2substituted quinolines in good yields (3h and $\mathbf{3 i}$ ). We then explored the heteroaromatic radical acceptor. Firstly, differently substituted quinolines were subjected to the optimized reaction conditions. Thus, methoxy- and bromide-bearing 4-methyl-substituted quinolines provided the desired alkylated heterocyclic systems (3j, 3k and $\mathbf{3} \mathbf{l})$. Notably, aromatic bromides were perfectly compatible with the electrochemical reaction conditions, which should be highlighted as the presence of bromides are very often circumvented in the substrate scope evaluation of previous Minisci-type alkylation studies using alkyl halides, ${ }^{[28,30-33]}$ probably to avoid other reduction byproducts. We then evaluated the substitution at the 4-position of the quinoline. In addition to a phenyl substituent ( $3 \mathrm{~m})$, elusive moieties in previous Minisci alkylative protocols such as bromide (30), ketone (3n), ester $(\mathbf{3} \mathbf{p})$ and amide $(\mathbf{3 q})^{[28,30-33]}$ were very well tolerated, giving rise to the alkylated quinolines with good yields. Additionally, we envisioned that 2-

methylquinolines could lead to the desired 4-alkylated products as well (3r-t). Consequently, various $N$ heteroarenes were tested, such as phenanthridine (3u), benzothiazole ( $\mathbf{3 v}$ ) and benzimidazole $(\mathbf{3} \mathbf{w})$, which were selectively alkylated at the most electrophilic position in good yields. Moreover, in order to extend the applicability of the method, other recognizable quinolines were also studied under these electrochemical conditions. Thus, late-stage alkylations of complex natural products decorated with various functionalities as quinoxyfen (fungicide, $\mathbf{3 x}$ ), quinine (3y), cinchonidine (3z) and $(S)-(+)$-Camptothecin (antitumor activity, 3aa) provided the corresponding $\mathrm{C} 2$ or $\mathrm{C} 4$ alkylation products in efficient fashion. Notably, the model reaction of lepidine (1a) proceeded efficiently starting from $1 \mathrm{mmol}$ (upscaling 10 times) and led to the desired alkylated quinoline derivative $\mathbf{3 a}$ in good yield $(75 \%$, see Supplementary Information).

With the idea to expand the applicability of the method, we identified acridines as potential substrates for their selective and straightforward alkylation at $\mathrm{C} 9$ by using the presented electrochemical methodology. Acridine derivatives constitute a class of compounds with a broad spectrum of biological activity and are of great interest for the organic and medicinal chemistry field.' Therefore, due to the lack of straightforward approaches to accomplish the direct functionalization of acridines in the literature, the synthetic modification of this prized heterocyclic core could be particularly appealing. Gratifyingly, under slightly modified reaction conditions (see Supplementary Information for details), acridine (1v) was found to be a suitable alkyl radical acceptor. Thus, the tert-butyl radical was added to the $\mathrm{C}-9$ position of the acridine, giving rise to the corresponding dihydroacridine derivative in good yields (Table $3,4 a$ ). 
We next examined the allylation and benzylation of acridine, which would give access to products whose synthesis, to the best of our knowledge, has never been accomplished by a Minisci-type alkylation protocol. Therefore, allyl bromide was efficiently reduced under the electrochemical reaction conditions and led to the desired allylated product $\mathbf{4 b}$ in good yield. Different benzyl bromides with varying electronic nature (4c-h) were tolerated using 2-MeTHF (included in the "green" solvent list) as an environmentally friendly solvent. Notably, synthetically versatile halides such as chloride (4d) and even iodides (4e) were compatible under the electrochemical reaction setup, leading to the benzylated dihydroacridine derivatives in good yields. Furthermore, the system was easily rearomatized under mild oxidative conditions to give the corresponding C9-functionalized acridine (5) in excellent yield.

As shown before, interesting and versatile carboxylic acid derivatives allocated at the 4-position of the quinoline were remarkably compatible under the electrochemical reaction conditions (Table 2). Encouraged by these results and in attempt to expand the applicability of the method, we targeted the formal synthesis of various interesting drug molecules. Thus, the iso-propyl installation at the 2 position of ester-bearing quinoline $1 \mathrm{~h}$ using our optimized electrochemical protocol led to the desired alkylated quinoline $\mathbf{6}$ with excellent selectivity. Simple hydrolysis of the ester moiety gave rise to carboxylic acid derivative 7, which has been used as a template for the construction of a more complex amidesubstituted quinoline featured as a glucose transport inhibitor (Scheme 1A). In addition, alkylated product 8, achieved directly following the electrochemical procedure, gave direct access to an intermediate in the synthesis of a tumor necrosis factor-a-converting enzyme (TACE) inhibitor (Scheme 1B). To our delight, this electrochemical system could also provide the corresponding alkylated quinoline 9 in the presence of a free carboxylic acid in synthetically useful yield, which is itself an intermediate in the synthesis of an antitumor agent (Scheme 1C) or used as template for the construction of anti-tuberculosis agents.

Mechanistic studies. We then performed a series of preliminary control experiments in order to gain an insight into the reaction mechanism. As shown in Scheme 2, in the presence of a radical scavenger as TEMPO (2,2,6,6-tetramethylpiperidinooxy), the reaction was inhibited as only starting materials were observed untouched (Scheme 2A). In addition, when an acrylamide derivative (10) was used as radical acceptor $^{41,47}$ instead of lepidine, the corresponding Giese-type product was obtained in good yield (11) (Scheme 2B). These results strongly indicate that radical species are involved in the reaction pathway. When the reaction under the standard conditions was carried out in absence of lepidine (1a), the alkyl halide was completely consumed, and only the homocoupling product, resulted from the cyclohexyl radical dimerization, was observed (Scheme $2 \mathrm{C}$ ). However, when performed in absence of $\mathbf{2 a}$, lepidine activated with PA did not suffer any change or reduction process (Scheme 2D), which shows how the optimized electrochemical reaction conditions are unable to reach the required reduction potential (with and without PA, see S.I.) for such transformation (see cyclic voltammetry Scheme 2). Therefore, the PA is only enhancing the reactivity of the $\mathrm{C}=\mathrm{N}$ bond. ${ }^{17}$ This may show a reductive pathway for the aliphatic halide, which might be generating carbon-centered radical species involved in the $\mathrm{C}$ - $\mathrm{C}$ bond forming event to yield the alkylated products. In addition, we tested the reaction under inert atmosphere conditions in order to evaluate if oxygen may be involved. In fact, under such conditions the reaction did not take place 
and only the starting materials were observed (Scheme 2E). Moreover, as expected based on the high reduction potential shown by halide $\mathbf{2 a}$ (see cyclic voltammetry Scheme 2), when a control experiment in absence of lepidine was carried out, no homocoupling product was observed (Scheme 2F). Therefore, oxygen should be involved in the generation of the initial reactive radical species. Moreover, as shown in Scheme $2 \mathrm{~A}$, in the presence of a superoxide scavenger as benzoquinone (BQ), the reaction was inhibited and the radical precursor was observed untouched.

Based on the above-mentioned findings and supported by the literature, ${ }^{47,40}$ we propose the mechanism shown on Scheme 2. We suggest that aerobic oxygen is responsible for the initiation of the process. Upon reduction, shown feasible with the lowest reduction potential of the reaction components (see cyclic voltammetry Scheme 2), the superoxide anion is formed and protonated to generate highly reactive peroxy radical species. These intermediates could be responsible for the generation of the carboncentered radical via halogen atom abstraction of the alkyl halide. Following alkyl radical generation, addition to the activated (protonated) $N$-heteroarene would result in the formation of a new carboncarbon bond. Finally, the putative radical intermediate would then undergo rearomatization to deliver the final Minisci-type adduct.

In conclusion, we have described a general and facile Minisci-type alkylation of $N$-heteroarenes under simple and straightforward electrochemical conditions using available alkyl halides as radical precursors. The reaction system has demonstrated its robustness and generality as primary, secondary and tertiary alkyl radical precursors and a variety of heterocycles have shown their compatibility within this electrochemical system. In addition, various heterocyclic-based natural products have been successfully integrated in the reaction scope. Moreover, as a consequence of the high functional group tolerance of the method, we have shown how the electrochemical Minisci-type alkylation methodology can be efficiently used to reach to biologically valuable compounds.

\section{Methods}

General electrochemical Minisci alkylation procedure. Diphenyl phosphate (25.0 mg, 1 equiv.), ammonium hexafluorophosphate $(81.5 \mathrm{mg}, 5$ equiv.) and a magnetic stirrer were added to a $5 \mathrm{~mL}$ ElectraSyn vial. Reagents were dissolved in THF $(2 \mathrm{~mL})$ and to the stirred solution were added $1(0.1$ $\mathrm{mmol}$ ) and 2 (5 equiv.), followed by water $(1 \mathrm{~mL})$. The vial was closed, reticulated vitreous carbon was used as working electrode and nickel foam as counter electrode, ElectraSyn 2.0 was set at constant current (10 mA) during $120 \mathrm{~min}$. The crude mixture was diluted with ethyl acetate, extracted with saturated aqueous solution of $\mathrm{NaHCO}_{3}(2 \times 5 \mathrm{~mL})$, washed with brine $(3 \times 30 \mathrm{~mL})$, dried over $\mathrm{MgSO}_{4}$, filtered and the solvent was evaporated under reduced pressure. The crude mixture was purified by column chromatography using silica gel and the eluent indicated in each case.

\section{Data availability}

The authors declare that all data supporting the findings of this study are available within the article and Supplementary Information files, and also are available from the corresponding author upon reasonable 
request.

\section{References}

1. Heravi, M. M.; Zadsirjan, V.; Prescribed drugs containing nitrogen heterocycles: an overview. RSC Adv., 10, 44247-44311 (2020).

2. Eicher, T.; Hauptmann, S.; Speicher, A. The Chemistry of Heterocycles, Wiley-VCH Verlag GmbH \& Co, Weinheim, 2nd edn, 2003.

3. Fujiwara, Y.; Baran, P. S. in Radical-Based Late Stage C-H Functionalization of Heteroaromatics in Drug Discovery. In New Horizons of Process Chemistry

4. Duncton, M. A. J.; Minisci reactions: Versatile $\mathrm{CH}$-functionalizations for medicinal chemists. Med. Chem. Commun., 2, 1135-1161 (2011).

5. Proctor, R. S. J.; Phipps, R. J. Recent Advances in Minisci-Type Reactions. Angew. Chem., Int. Ed., 58, 13666-13699 (2019).

6. Tomioka, K., Shioiri, T., Sajiki, H., Eds.; Springer Nature: Singapore, 2017; pp 103-120

7. DiRocco, D. A.; Dykstra, K.; Krska, S.; Vachal, P.; Conway D. V.; Tudge, M. Late-Stage Functionalization of Biologically Active Heterocycles Through Photoredox Catalysis. Angew. Chem., Int. Ed., 53, 48024806 (2014).

8. Jin, J.; MacMillan, D. W. C. Alcohols as alkylating agents in heteroarene C-H functionalization. Nature, 525, 87-90 (2015).

9. Li, G.-X.; Morales-Rivera, C. A.; Wang, Y.; Gao, F.; He, G.; Liu, P.; Chen, G. Photoredox-mediated Minisci $\mathrm{C}-\mathrm{H}$ alkylation of $\mathrm{N}$-heteroarenes using boronic acids and hypervalent iodine. Chem. Sci., 7, 64076412 (2016).

10. Dong, J.; Yue, F.; Song, H.; Liu, Y.; Wang, Q. Visible-light-mediated photoredox minisci C-H alkylation with alkyl boronic acids using molecular oxygen as an oxidant. Chem. Commun., 56, 12652-12655 (2020).

11. Matsui, J. K.; Primer, D. N.; Molander, G. A. Metal-free C-H alkylation of heteroarenes with alkyltrifluoroborates: a general protocol for $1^{\circ}, 2^{\circ}$ and $3^{\circ}$ alkylation. Chem. Sci., 8, 3512-3522 (2017).

12. Garza-Sanchez, R. A.; Tlahuext-Aca, A.; Tavakoli, G.; Glorius, F. Visible Light-Mediated Direct Decarboxylative C-H Functionalization of Heteroarenes. ACS Catal., 7, 4057-4061 (2017).

13. Wang, J.; Li, G.-X.; He, G.; Chen, G. Photoredox-Mediated Minisci Alkylation of N-Heteroarenesusing Carboxylic Acids and Hypervalent lodine. Asian J. Org. Chem., 7, 1307-1310 (2018).

14. Tian, W. -F.; Hu, C. -H.; He, K. -H.; He, X. -Y.; Li, Y. Visible-Light Photoredox-Catalyzed Decarboxylative Alkylation of Heteroarenes Using Carboxylic Acids with Hydrogen Release. Org. Lett., 21, 6930-6935 (2019).

15. Papaioannou, N.; Fray, M. J.; Rennhack, A.; Sanderson, T. J.; Stokes, J. E. Regioselective Amidomethylation of 4-Chloro-3-fluoropyridine by Metalation and Minisci-Type Reactions. J. Org. Chem., 85, 12067-12079 (2020). 
16. For a selected review, see: Parida, S. K.; Mandal, T.; Das, S.; Hota, S. K.; Sarkar, S. D.; Murarka, S. Single Electron Transfer-Induced Redox Processes Involving N-(Acyloxy)phthalimides. ACS Catal., 11, 1640-1683 (2021).

17. Jin, S.; Geng, X.; Li, Y.; Zheng, K. Phosphoric Acid Mediated Light-Induced Minisci C-H Alkylation of NHeteroarenes. Eur. J. Org. Chem., 969-972 (2021).

18. Hagui, W.; Cordier, M.; Boixel, J.; Soulé, J. -F. Access to functionalized luminescent Pt(II) complexes by photoredox-catalyzed Minisci alkylation of 6-aryl-2,20-bipyridines. Chem. Commun., 57, 1038-1041 (2021).

19. Dong, J.; Wang, X.; Song, H.; Liu, Y.; Wang. Q. Photoredox-Catalyzed Redox-Neutral Minisci C-H Formylation of N-Heteroarenes. Adv. Synth. Catal. 362, 2155-2159 (2020).

20. Qin, P. -T.; Sun, J.; Wang, F.; Wang, J. -Y.; Wang, H.; Zhoua, M. -D. Visible-Light-Induced C2 Alkylation of Heterocyclic N-Oxides with N-Hydroxyphthalimide Esters under Metal-Free Conditions. Adv. Synth. Catal., 362, 4707-4715 (2020).

21. Capaldo, L.; Quadri, L. L.; Merli D.; Ravelli, D. Photoelectrochemical cross-dehydrogenative coupling of benzothiazoles with strong aliphatic C-H bonds. Chem. Commun., 57, 4424-4427 (2021).

22. Proctor, R. S. J.; Chuentragool, P.; Colgan, A. C.; Phipps, R. J. Hydrogen Atom Transfer-Driven Enantioselective Minisci Reaction of Amides. J. Am. Chem. Soc., 143, 4928-4934 (2021)

23. Deng, Z.; Li, G. -X.; He, G.; Chen, G. Photoredox-Mediated Remote C(sp3)-H Heteroarylation of N-Alkyl Sulfonamides. J. Org. Chem., 84, 15777-15787 (2019).

24. Wang, Z.; Ji, X.; Han, T.; Deng, G. -J.; Huang, H. LiBr-Promoted Photoredox Minisci-Type Alkylations of Quinolines with Ethers. Adv. Synth. Catal., 361, 5643-5647 (2019).

25. Tian, H.; Yang, H.; Tian, C.; An, G.; Li, G. Cross-Dehydrogenative Coupling of Strong C(sp3)-H with N-Heteroarenes through Visible-Light-Induced Energy Transfer. Org. Lett., 22, 7709-7715 (2020).

26. Rammal, F.; Gao, D.; Boujnah, S.; Gaumont, A. -C.; Hussein, A. A.; Lakhdar, S. Visible-Light-Mediated C-H Alkylation of Pyridine Derivatives. Org. Lett., 22, 7671-7675 (2020).

27. Li, G.; Gao, Y.; Jia, C.; Wang, S.; Yan, B.; Fang, Y.; Yang, S. Meta-Dehydrogenative Alkylation of Arenes with Ethers, Ketones, and Esters Catalyzed by Ruthenium. Org. Lett., 22, 8758-8763 (2020).

28. Santos, M. S.; Cybularczyk-Cecotka, M.; König, B.; Giedyk, M. Minisci C-H Alkylation of Heteroarenes Enabled by Dual Photoredox/Bromide Catalysis in Micellar Solutions. Chem. Eur. J., 26, 15323-15329 (2020).

29. Jung, S.; Shin, S.; Park, S.; Hong, S. Visible-Light-Driven C4-Selective Alkylation of Pyridinium Derivatives with Alkyl Bromides. J. Am. Chem. Soc., 142, 11370-11375 (2020).

30. Dong, J.; Lyu, X.; Wang, Z.; Wang, X.; Song, H.; Liua, Y.; Wang, Q. Visible-light-mediated Minisci C-H alkylation of heteroarenes with unactivated alkyl halides using $\mathrm{O}_{2}$ as an oxidant. Chem. Sci., 10, 976982. (2019).

31. Perkins, J. J.; Schubert, J. W.; Streckfuss, E. C.; Balsells, J.; ElMarrouni, A. Photoredox Catalysis for Silyl-Mediated C-H Alkylation of Heterocycles with Non-Activated Alkyl Bromides. Eur. J. Org. 
Chem., 1515-1522 (2020).

32. Nuhant, P.; Oderinde, M. S.; Genovino, J.; Juneau, A.; Gagné, Y.; Allais, C.; Chinigo, G. M.; Choi, C.; Sach, N. W.; Bernier, L.; Fobian, Y. M.; Bundesmann, M. W.; Khunte, B.; Frenette, M.; Fadeyi, O. O. Visible-LightInitiated Manganese Catalysis for C-H Alkylation of Heteroarenes: Applications and Mechanistic Studies. Angew. Chem. Int. Ed., 56, 15309-15313 (2017).

33. Bissonnette, N. B.; Boyd, M. J.; May, G. D.; Giroux, S.; Nuhant, P. C-H Functionalization of Heteroarenes Using Unactivated Alkyl Halides through Visible-Light Photoredox Catalysis under Basic Conditions. J. Org. Chem., 83, 10933-10940 (2018).

34. Möhle, S.; Zirbes, M.; Rodrigo, E.; Gieshoff, T.; Wiebe, A.; Waldvogel, S. R. Modern Electrochemical Aspects for the Synthesis of Value-Added Organic Products. Angew. Chem. Int. Ed., 57, 60186041 (2018)

35. Meyer, T. H.; Choi, I.; Tian, C.; Ackermann, L. Powering the Future: How Can Electrochemistry Make a Difference in Organic Synthesis? Chem, 6, 1-13 (2020).

36. Yan, M.; Kawamata, Y.; Baran, P. S. Synthetic Organic Electrochemical Methods Since 2000: On the Verge of a Renaissance. Chem. Rev., 117, 13230-13319 (2017).

37. Horn, E. J.; Rosen, B. R.; Baran, P. S. Synthetic Organic Electrochemistry: An Enabling and Innately Sustainable Method. ACS Cent. Sci., 2, 302-308 (2016).

38. Pollok, D.; Waldvogel, S. R. Electro-organic synthesis - a 21 st century technique. Chem. Sci., 11, 12386-12400 (2020).

39. O'Brien, A. G.; Maruyama, A.; Inokuma, Y.; Fujita, M.; Baran, P. S. Radical C-H Functionalization of Heteroarenes under Electrochemical Control. Angew. Chem., Int. Ed., 53, 11868-11871 (2014)

40. Liu, Y.; Xue, L.; Shi, B.; Bu, F.; Wang, D.; Lu, L.; Shi, R.; Lei, A. Catalyst-free electrochemical decarboxylative cross-coupling of $\mathrm{N}$-hydroxyphthalimide esters and $\mathrm{N}$-heteroarenes towards $\mathrm{C}(\mathrm{sp} 3)$ C(sp2) bond formation. Chem. Commun., 55, 14922-14925 (2019).

41. Niu, K.; Song, L.; Hao, Y.; Liu, Y.; Wang, Q. Electrochemical decarboxylative C3 alkylation of quinoxalin2(1H)-ones with N-hydroxyphthalimide esters. Chem. Commun., 56, 11673-11676 (2020).

42. Gao, Y.; Wu, Z.; Yu, L.; Wang, Y.; Pan, Y. Alkyl Carbazates for Electrochemical Deoxygenative Functionalization of Heteroarenes. Angew. Chem., Int. Ed., 59, 10859-10863 (2020).

43. Yang, D. -T.; Zhu, M.; Schiffer, Z. J.; Williams, K.; Song, X.; Liu, X.; Manthiram., K. Direct Electrochemical Carboxylation of Benzylic C-N Bonds with Carbon Dioxide. ACS Catal., 9 , 4699-4705 (2019)

44. Huang, J. -M.; Wang, X. -X.; Dong. Y. Electrochemical Allylation Reactions of Simple Imines in Aqueous Solution Mediated by Nanoscale Zinc Architectures. Angew. Chem. Int. Ed., 50, 924-927 (2011).

45. Huang, B.; Guo, L.; Xia, W. A facile and versatile electro-reductive system for hydrodefunctionalization under ambient conditions. Green Chem., 23, 2095-2103 (2021). 
46. Lian, F.; Xu, K.; Meng, W.; Zhang, H.; Tana, Z.; Zeng, C. Nickel-catalyzed electrochemical reductive decarboxylative coupling of N-hydroxyphthalimide esters with quinoxalinones. Chem. Commun., 55, 14685-14688 (2019).

47. Li, D.; Ma, T. -K.; Scott R. J.; Wilden, J. D. Electrochemical radical reactions of alkyl iodides: a highly efficient, clean, green alternative to tin reagents. Chem. Sci., 11, 5333-5338 (2020).

48. Paddon, C. A.; Bhatti, F. L.; Donohoe, T. J.; Compton, R. G. Electrocatalytic reduction of alkyl iodides in tetrahydrofuran at silver electrodes. J. Phys. Org. Chem., 20, 115-121 (2007).

49. Gensicka-Kowalewska, M.; Cholewinski, G.; Dzierzbicka, K. Recent developments in the synthesis and biological activity of acridine/acridone analogues. RSC Adv., 7, 15776-15804 (2017).

50. Gabriel, I. 'Acridines' as New Horizons in Antifungal Treatment. Molecules, 25, 1480-1495 (2020)

51. For a Minisci benzylation protocol, see: Dong, J.; Wang, X.; Wang, Z.; Song, H.; Liu Y.; Wang, Q. Metal-, photocatalyst-, and light-free late-stage $\mathrm{C}-\mathrm{H}$ alkylation of $\mathrm{N}$-heteroarenes with organotrimethylsilanes using persulfate as a stoichiometric oxidant. Org. Chem. Front., 6, 2902-2906 (2019).

52. Heisler, I; Müller, T.; Siebeneicher, H.; Buchmann, B.; Cleve, A.; Günther, J.; et al. Glucose transport inhibitors. W02015091428

53. Argade, A.; Bahekar, R.; Desai, J.; Thombare, P.; Shah, K.; Gite, S. et al. Design, synthesis and biological evaluation of g-lactam hydroxamate based TACE inhibitors. Med. Chem. Commun., 2, 966-972 (2011).

54. Chen, X.; Sun, W.; Huang, S.; Zhang, H.; Lin, G.; Li, H.; Qiao, J.; Li, L.; Yang, S. Discovery of Potent Small-Molecule SIRT6 Activators: Structure- Activity Relationship and Anti-Pancreatic Ductal Adenocarcinoma Activity. J. Med. Chem., 63, 10474-10495 (2020).

55. Nayyar, A.; Malde, A.; Jain, R.; Coutinho, E. 3D-QSAR study of ring-substituted quinoline class of antituberculosis agents. Bioorganic \& Medicinal Chemistry, 14, 847-856 (2006).

\section{Declarations}

\section{Acknowledgements}

Financial support was provided by the European Research Council (ERC-CoG-647550), Spanish Government (RTI2018-095038-B-I00), "Comunidad de Madrid" for European Structural Funds (S2018/NMT-4367) and proyectos sinergicos I+D (Y2020/NMT-6469). J. A. F.-S. thanks the Spanish Government for a Ramón y Cajal contract. R. del Río-Rodríguez thanks the "Comunidad de Madrid" for a predoctoral fellowship.

\section{Author contributions}

R.R.R. carried out the optimization and scope of the reaction. L.F and A.F.G.C participated in the optimization. M.C.M., J.A.F.S and J.A. conceived the project and prepared the manuscript, which was edited by all other authors. 
Competing financial interests

The authors declare no competing interests.

\section{Tables}

Tables 1-3 are available in the Supplemental Files section

\section{Schemes}

Schemes 1 and 2 are available in the Supplemental File section

\section{Figures}

\section{A. Electrochemical Minisci-type alkylation}

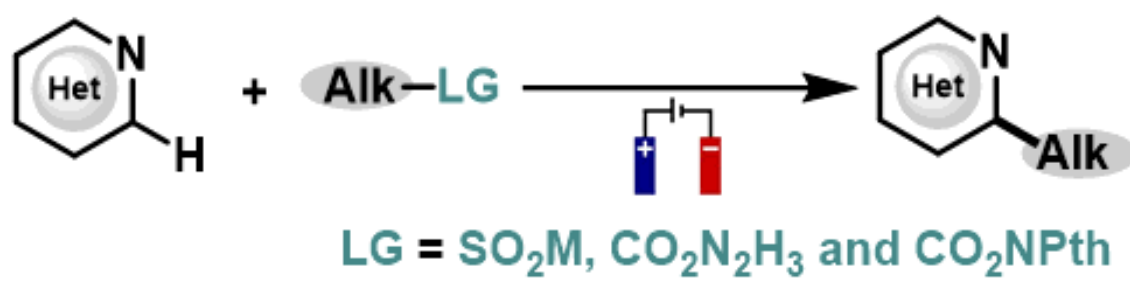

B. This work. Electrochemical Minisci alkylation of $\mathrm{N}$-heteroarenes with alkyl halides
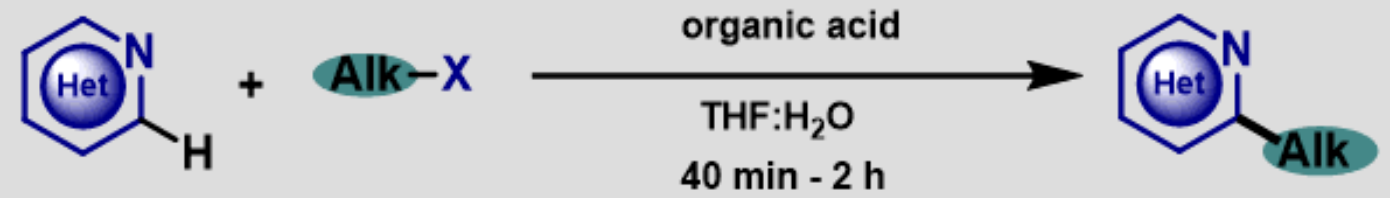

\section{Figure 1}

Previous work. A: Electrochemical Minisci-type alkylation. B: This work. Electrochemical Minisci alkylation of $N$-heteroarenes with alkyl halides.

\section{Supplementary Files}

This is a list of supplementary files associated with this preprint. Click to download.

- Onlinefloatimage2.png

- Onlinefloatimage3.png

- Onlinefloatimage4.png 
- Onlinefloatimage6.png

- Onlinefloatimage7.png

- Onlinefloatimage5.png

- Supportinginformation.pdf 